\title{
Dynamic behavior of pipes conveying gas-liquid two-phase flow
}

\author{
Chen $\mathrm{An}^{\mathrm{a}}$, Jian $\mathrm{Su}^{\mathrm{b}, *}$ \\ a Offshore Oil/Gas Research Center, China University of Petroleum-Beijing, Beijing 102249, China \\ ${ }^{\mathrm{b}}$ Nuclear Engineering Program, COPPE, Universidade Federal do Rio de Janeiro, CP 68509, Rio de Janeiro 21941-972, Brazil
}

\section{H I G H L I G H T S}

- Dynamic behavior of pipes conveying gas-liquid two-phase flow was analyzed.

- The generalized integral transform technique (GITT) was applied.

- Excellent convergence behavior and long-time stability were shown.

- Effects of volumetric quality and volumetric flow rate on dynamic behavior were studied.

- Normalized volumetric-flow-rate stability envelope of dynamic system was determined.

\section{A R T I C L E I N F O}

\section{Article history:}

Received 13 October 2014

Received in revised form 23 June 2015

Accepted 25 June 2015

\begin{abstract}
A B S T R A C T
In this paper, the dynamic behavior of pipes conveying gas-liquid two-phase flow was analytically and numerically investigated on the basis of the generalized integral transform technique (GITT). The use of the GITT approach in the analysis of the transverse vibration equation lead to a coupled system of second order differential equations in the dimensionless temporal variable. The Mathematica's built-in function, NDSolve, was employed to numerically solve the resulting transformed ODE system. The characteristics of gas-liquid two-phase flow were represented by a slip-ratio factor model that was devised and used for similar problems. Good convergence behavior of the proposed eigenfunction expansions is demonstrated for calculating the transverse displacement at various points of pipes conveying air-water two-phase flow. Parametric studies were performed to analyze the effects of the volumetric gas fraction and the volumetric flow rate on the dynamic behavior of pipes conveying air-water two-phase flow. Besides, the normalized volumetric-flow-rate stability envelope for the dynamic system was obtained.
\end{abstract}

(c) 2015 Elsevier B.V. All rights reserved.

\section{Introduction}

Internal flow-induced structural vibration has been experienced in numerous fields, including heat exchanger tubes, chemical plant piping systems, nuclear reactor components and subsea production pipelines. The systematic and extensive investigations have been carried out in the past decades to understand the dynamic behavior of pipes conveying single-phase flow (Païdoussis and Li, 1993; Païdoussis, 1998, 2008; Kuiper and Metrikine, 2005). Recently, many achievements have been made in understanding the dynamic characteristics of pipes conveying fluid. Kang (2000) investigated the effects of rotary inertia of concentrated masses on the free vibrations and system instability of the clamped-supported pipe conveying fluid, and concluded that introduction of rotary

\footnotetext{
* Corresponding author. Tel.: +55 213938 8448; fax: +55 2139388444.

E-mail addresses: anchen@cup.edu.cn (C. An), sujian@lasme.coppe.ufrj.br (J.Su).
}

inertia can influence the higher natural frequencies and mode shapes of fluid-conveying pipes. Sinha et al. (2005) utilized a nonlinear optimization method to predict the flow-induced excitation forces and the structural responses of the piping system, whose validation was presented through a simulated experiment on a long straight pipe conveying fluid. Based on the principle of eliminated element-Galerkin method, Huang et al. (2010) reduced the binary partial differential equation to an ordinary differential equation using the separation of variables, then utilized Galerkin method to obtain the natural frequency equations of pipeline conveying fluid with different boundary conditions, and found that the effect of Coriolis force on natural frequency is inappreciable. Li et al. (2011) presented a Timoshenko beam theory-based model for the dynamics of pipes conveying fluid, and proposed the dynamic stiffness method to analyze the free vibration of multi-span pipe. Zhai et al. (2011) determined the natural frequencies of fluid-conveying Timoshenko pipes via the complex modal analysis, and calculated the dynamic response of pipeline under random excitation by 
the pseudo excitation algorithm. Zhang and Chen (2012) analyzed the internal resonance of pipes conveying fluid in the supercritical regime, in which the straight pipe equilibrium configuration becomes unstable and bifurcates into two possible curved equilibrium configurations. As the extension of the previous work, Zhang and Chen (2013) and Chen et al. (2014) studied the nonlinear forced vibration of a viscoelastic pipe conveying fluid around the curved equilibrium configuration resulting from the supercritical flow speed, where the frequency and amplitude relationships of stead-state responses in external and internal resonances were derived.

However, the literature on the dynamic behavior of the pipes subjected to two-phase internal flow, which commonly occurs in piping elements, is still sparse. A few studies have been conducted to investigate the vibration behavior of pipes conveying gas-liquid two-phase flow. Hara (1977) performed the experimental and theoretical analyses of the excitation mechanism of air-water two-phase flow induced vibrations of a straight horizontal pipe, and found that vibrations were dominantly excited by parametric excitation and by natural vibration resonance to the external force through the instability analysis of a system of Mathieu equations. Fluidelastic instability occurs at a critical velocity for which the energy from the fluid is no longer dissipated entirely by the damping of the piping system (Riverin and Pettigrew, 2007), which is the most important of several flow-induced vibration excitation mechanisms that can cause large oscillations and early pipe failure. Monette and Pettigrew (2004) reported the results of a series of experiments to study the fluidelastic instability behavior of cantilevered flexible tubes subjected to two-phase internal flow, where a modified two-phase model was proposed to formulate the characteristics of two-phase flows. Pettigrew and Taylor (1994) outlined cylinders experienced fluidelastic instabilities in liquid flow in the form of buckling or oscillations, and fluidelastic instabilities can be affected by several parameters such as flow velocity, void fraction, flexural rigidity, end conditions and annular confinement. Pettigrew et al. (1998) analyzed the relative importance of flow-induced vibration excitation mechanisms (fluidelastic instability, periodic wake shedding, turbulence-induced excitation and acoustic resonance) for liquid, gas and two-phase axial flow. Riverin et al. (2006) measured the time-dependent forces resulting from a two-phase air-water mixture flowing in an elbow and a tee, and explored a relation between the fluctuating forces caused by the two-phase flow and the characteristics of the flow. Riverin and Pettigrew (2007) conducted an experimental study to investigate the governing vibration excitation mechanism governing in-plane vibrations observed on U-shaped piping elements. Zhang and $\mathrm{Xu}$ (2010) carried out an experimental study of pipe vibrations subjected to internal bubbly flow. Zhang et al. (2010) examined the characteristics of channel wall vibration induced by internal bubbly flow, and developed a mathematical model that can well predicted the spectral frequencies of the wall vibrations and pressure fluctuations, the corresponding attenuation coefficients and propagation phase speeds. Recently, flow-induced vibration of pipelines conveying high-pressure oil and gas has received attention since it causes damage to supporting structures or pipeline ruptures leading to costly shutdown and severe environmental problems Guo et al. (2014). Patel and Seyed (1989) examined the contribution of time varying internal slug flow to the dynamic excitation forces applied to a flexible riser. Seyed and Patel (1992) deduced an analytical model for calculating the pressure and internal slug flow induced forces on flexible risers. Ortega et al. (2012) reported a computational method to analyze the interaction between an internal slug flow and the dynamic response of flexible risers. Extending previous work, Ortega et al. (2013) studied the interaction between an internal slug flow plus an external regular wave and the dynamic response of a flexible riser.
In this study, the dynamic behavior of pipes conveying gas-liquid two-phase flow is analytically and numerically investigated on the basis of the generalized integral transform technique (GITT), which has been successfully developed in heat and fluid flow applications (Cotta, 1993, 1998; Cotta and Mikhailov, 1997) and further applied in solving the dynamic response of axially moving beams (An and Su, 2011), axially moving Timoshenko beams (An and Su, 2014b), axially moving orthotropic plates (An and Su, 2014a), damaged Euler-Bernoulli beams (Matt, 2013a), cantilever beams with an eccentric tip mass (Matt, 2013b), fluidconveying pipes (Gu et al., 2013), the wind-induced vibration of overhead conductors (Matt, 2009) and the vortex-induced vibration of long flexible cylinders (Gu et al., 2012). The most interesting feature in this technique is the automatic and straightforward global error control procedure, which makes it particularly suitable for benchmarking purposes, and the only mild increase in overall computational effort with increasing number of independent variables. The main contribution is the application of the effective tool, the generalized integral transform technique, to developing an analytical-numerical approach to determine the dynamic characteristics of pipes conveying gas-liquid two-phase flow, aiming at providing more reference data in this topic for future comparison.

The rest of paper is organized as follows: in the next section, the mathematical formulation of the transverse vibration problem of pipes conveying gas-liquid two-phase flow is presented. In Section 3, the hybrid numerical-analytical solution is obtained by carrying out integral transform. Numerical results of proposed method including transverse displacements and their corresponding convergence behavior are presented in Section 4. A parametric study is then performed to investigate the effects of the volumetric qualities on natural frequencies and vibration amplitudes of pipes conveying air-water two-phase flow, respectively. Besides, the volumetric-flow-rate stability envelope for the system is also presented. Finally, the paper ends in Section 5 with conclusions and perspectives.

\section{Mathematical formulation}

We consider a vertical pipe with clamped-clamped boundary conditions subjected to gas-liquid two-phase internal flow, as illustrated in Fig. 1, where the internal flow of the pipe is constituted by the discrete gaseous bubbles in a continuous liquid. If internal damping, external imposed tension and pressurization effects are either absent or neglected, the equation of motion of the pipe can be derived following the Newtonian derivation by means of decomposing an infinitesimal pipe-fluid element into the pipe element and the fluid element under the assumptions of the Euler-Bernoulli beam theory, according to the procedures given by Païdoussis (1998) and Monette and Pettigrew (2004):

$$
\begin{aligned}
E I \frac{\partial^{4} w}{\partial x^{4}} & +\sum_{k} M_{k} U_{k}^{2} \frac{\partial^{2} w}{\partial x^{2}}+2 \sum_{k} M_{k} U_{k} \frac{\partial^{2} w}{\partial x \partial t}+\left(\sum_{k} M_{k}+m\right) \frac{\partial^{2} w}{\partial t^{2}} \\
& +\left(\sum_{k} M_{k}+m\right) g\left((x-L) \frac{\partial^{2} w}{\partial x^{2}}+\frac{\partial w}{\partial x}\right)=0
\end{aligned}
$$

subjected to the clamped-clamped boundary conditions

$w(0, t)=0, \quad \frac{\partial w(0, t)}{\partial x}=0, \quad w(L, t)=0, \quad \frac{\partial w(L, t)}{\partial x}=0$,

where $w(x, t)$ is the transverse displacement, EI is the flexural rigidity of the pipe which depends upon both Young's modulus $E$ and the inertial moment of cross-section area $I, M_{k}$ and $U_{k}$ are 


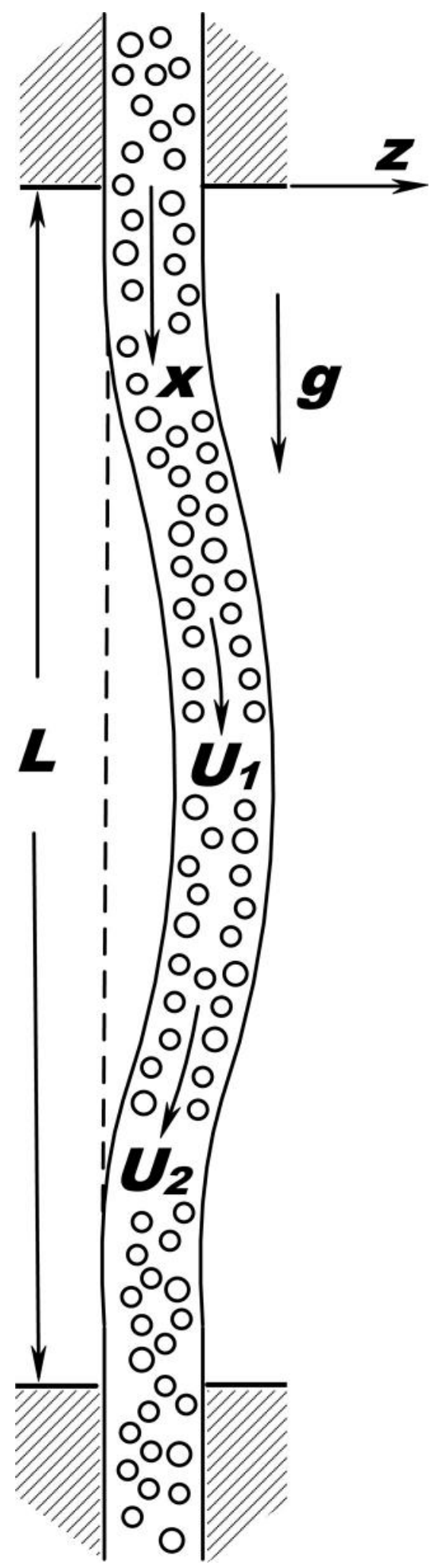

Fig. 1. Illustration of a clamped-clamped pipe conveying gas-liquid two-phase flow, where $U_{1}$ and $U_{2}$ express the velocities of the gas and the liquid phases.

respectively the masses per unit length and the steady flow velocities of the gas and the liquid phases ( $k$ takes the values of 1 for gas $(G)$ and 2 for liquid $(L)), m$ is the mass of the pipe per unit length, $L$ is the pipe length, and $g$ is the gravitational acceleration. Note that the terms of Eq. (1a) represents respectively: flexural force, centrifugal force, Coriolis force, inertia force and gravity, where centrifugal and Coriolis forces are generated by both gas and liquid phases. The following dimensionless variables are introduced

$$
\begin{aligned}
& x^{*}=\frac{x}{L}, \quad w^{*}=\frac{w}{L}, \quad \tau=\frac{t}{L^{2}} \sqrt{\frac{E I}{\sum_{k} M_{k}+m}}, \\
& \Gamma_{k}=U_{k} L \sqrt{\frac{M_{k}}{E I}}, \quad \beta_{k}=\frac{M_{k}}{\sum_{k} M_{k}+m}, \quad \gamma=g L^{3} \frac{\sum_{k} M_{k}+m}{E I} .
\end{aligned}
$$

Substituting Eq. (2) into Eq. (1) gives the dimensionless equation (dropping the superposed asterisks for simplicity)

$$
\begin{aligned}
\frac{\partial^{4} w}{\partial x^{4}} & +\sum_{k} \Gamma_{k}^{2} \frac{\partial^{2} w}{\partial x^{2}}+2 \sum_{k} \Gamma_{k} \beta_{k}^{1 / 2} \frac{\partial^{2} w}{\partial x \partial \tau}+\frac{\partial^{2} w}{\partial \tau^{2}} \\
& +\gamma\left((x-1) \frac{\partial^{2} w}{\partial x^{2}}+\frac{\partial w}{\partial x}\right)=0
\end{aligned}
$$

together with the boundary conditions

$w(0, \tau)=0, \quad \frac{\partial w(0, \tau)}{\partial x}=0, \quad w(1, \tau)=0, \quad \frac{\partial w(1, \tau)}{\partial x}=0$,

The initial conditions are defined as follows:

$w(x, 0)=0, \quad \dot{w}(x, 0)=v_{0} \sin (\pi x)$,

where the sign ' ' denotes the time derivative of the dimensionless transverse displacement.

\section{Integral transform solution}

According to the principle of the generalized integral transform technique, the auxiliary eigenvalue problem needs to be chosen for the dimensionless governing equation (3a) with the homogenous boundary conditions ( $3 b-e)$. The spatial coordinate ' $x$ ' is eliminated through integral transformation, and the related eigenvalue problem is adopted for the transverse displacement representation as follows:

$\frac{\mathrm{d}^{4} X_{i}(x)}{\mathrm{d} x^{4}}=\mu_{i}^{4} X_{i}(x), \quad 0<x<1$,

with the following boundary conditions

$X_{i}(0)=0, \quad \frac{\mathrm{d} X_{i}(0)}{\mathrm{d} x}=0$,

$X_{i}(1)=0, \quad \frac{\mathrm{d} X_{i}(1)}{\mathrm{d} x}=0$,

where $X_{i}(x)$ and $\mu_{i}$ are, respectively, the eigenfunctions and eigenvalues of problem (5). The eigenfunctions satisfy the following orthogonality property

$\int_{0}^{1} X_{i}(x) X_{j}(x) \mathrm{d} x=\delta_{i j} N_{i}$,

with $\delta_{i j}=0$ for $i \neq j$, and $\delta_{i j}=1$ for $i=j$. The norm, or normalization integral, is written as

$N_{i}=\int_{0}^{1} X_{i}^{2}(x) \mathrm{d} x$. 
Table 1

Convergence behavior of the dimensionless transverse displacement $w(x, \tau)$ of a pipe conveying air-water two-phase flow.

\begin{tabular}{|c|c|c|c|c|c|c|}
\hline$x$ & $N W=4$ & $N W=8$ & $N W=12$ & $N W=16$ & $N W=20$ & $N W=24$ \\
\hline \multicolumn{7}{|l|}{$\tau=5$} \\
\hline 0.1 & 0.00127 & -0.00676 & -0.00498 & -0.00468 & -0.00462 & -0.00460 \\
\hline 0.3 & 0.0121 & -0.0479 & -0.0360 & -0.0340 & -0.0336 & -0.0334 \\
\hline 0.5 & 0.0233 & -0.102 & -0.0770 & -0.0729 & -0.0719 & -0.0716 \\
\hline 0.7 & 0.0226 & -0.112 & -0.0873 & -0.0832 & -0.0822 & -0.0819 \\
\hline 0.9 & 0.00560 & -0.0258 & -0.0213 & -0.0206 & -0.0204 & -0.0204 \\
\hline \multicolumn{7}{|c|}{$\tau=20$} \\
\hline 0.1 & 0.00516 & 0.00756 & 0.0105 & 0.0103 & 0.0103 & 0.0102 \\
\hline 0.3 & 0.0298 & 0.0504 & 0.0768 & 0.0773 & 0.0772 & 0.0772 \\
\hline 0.5 & 0.0703 & 0.0855 & 0.157 & 0.161 & 0.161 & 0.162 \\
\hline 0.7 & 0.0892 & 0.0552 & 0.136 & 0.142 & 0.143 & 0.143 \\
\hline 0.9 & 0.0228 & 0.00682 & 0.0216 & 0.0227 & 0.0230 & 0.0231 \\
\hline \multicolumn{7}{|c|}{$\tau=50$} \\
\hline 0.1 & 0.00673 & -0.000719 & -0.00826 & -0.00673 & -0.00623 & -0.00604 \\
\hline 0.3 & 0.0569 & 0.00513 & -0.0705 & -0.0613 & -0.0583 & -0.0573 \\
\hline 0.5 & 0.134 & 0.0396 & -0.157 & -0.146 & -0.141 & -0.140 \\
\hline 0.7 & 0.121 & 0.0685 & -0.147 & -0.143 & -0.139 & -0.138 \\
\hline 0.9 & 0.0215 & 0.0170 & -0.0257 & -0.0259 & -0.0256 & -0.0254 \\
\hline
\end{tabular}

Problem (5) is readily solved analytically to yield

$X_{i}(x)= \begin{cases}\frac{\cos \left[\mu_{i}(x-1 / 2)\right]}{\cos \left(\mu_{i} / 2\right)}-\frac{\cosh \left[\mu_{i}(x-1 / 2)\right]}{\cosh \left(\mu_{i} / 2\right)}, \text { for } i \text { odd }, \\ \frac{\sin \left[\mu_{i}(x-1 / 2)\right]}{\sin \left(\mu_{i} / 2\right)}-\frac{\sinh \left[\mu_{i}(x-1 / 2)\right]}{\sinh \left(\mu_{i} / 2\right)}, \text { for } i \text { even }\end{cases}$

where the eigenvalues are obtained from the transcendental equations:

$\tanh \left(\mu_{i} / 2\right)= \begin{cases}-\tan \left(\mu_{i} / 2\right), & \text { for } i \text { odd } \\ \tan \left(\mu_{i} / 2\right), & \text { for } i \text { even }\end{cases}$

and the normalization integral is evaluated as

$N_{i}=1, \quad i=1,2,3, \ldots$

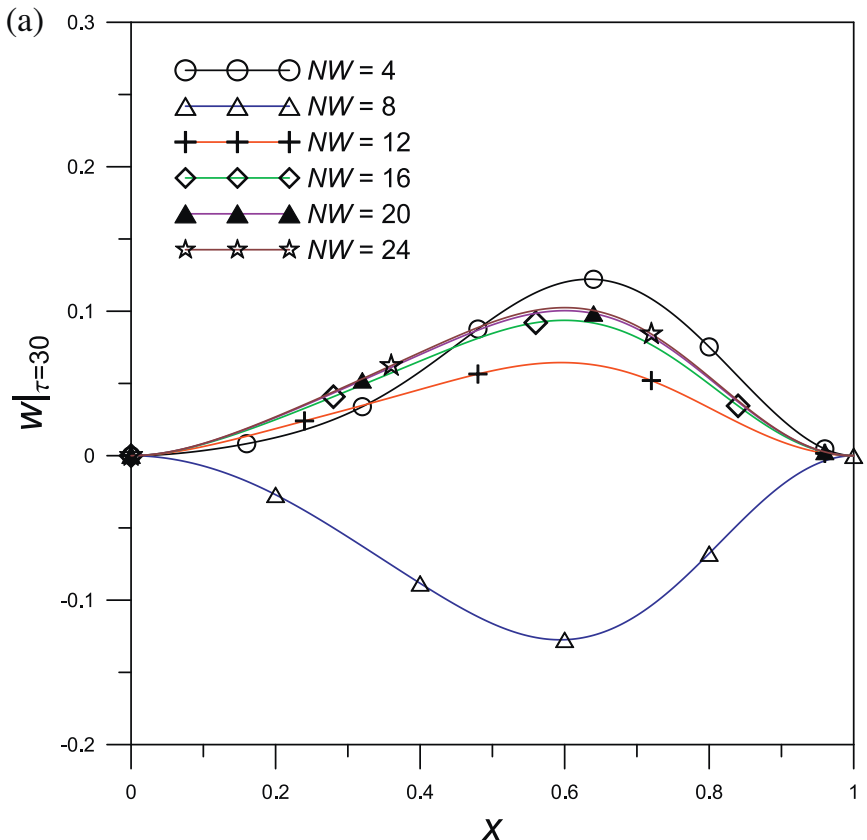

Therefore, the normalized eigenfunction coincides, in this case, with the original eigenfunction itself, i.e.

$\tilde{X}_{i}(x)=\frac{X_{i}(x)}{N_{i}^{1 / 2}}$.

The solution methodology proceeds towards the proposition of the integral transform pair for the potentials, the integral transformation itself and the inversion formula. For the transverse displacement:

$\bar{w}_{i}(\tau)=\int_{0}^{1} \tilde{X}_{i}(x) w(x, \tau) \mathrm{d} x, \quad$ transform,

$w(x, \tau)=\sum_{i=1}^{\infty} \tilde{X}_{i}(x) \bar{w}_{i}(\tau), \quad$ inverse.

The integral transformation process is now employed through operation of (3a) with $\int_{0}^{1} \tilde{X}_{i}(x) \mathrm{d} x$, to find the transformed transverse displacement system:

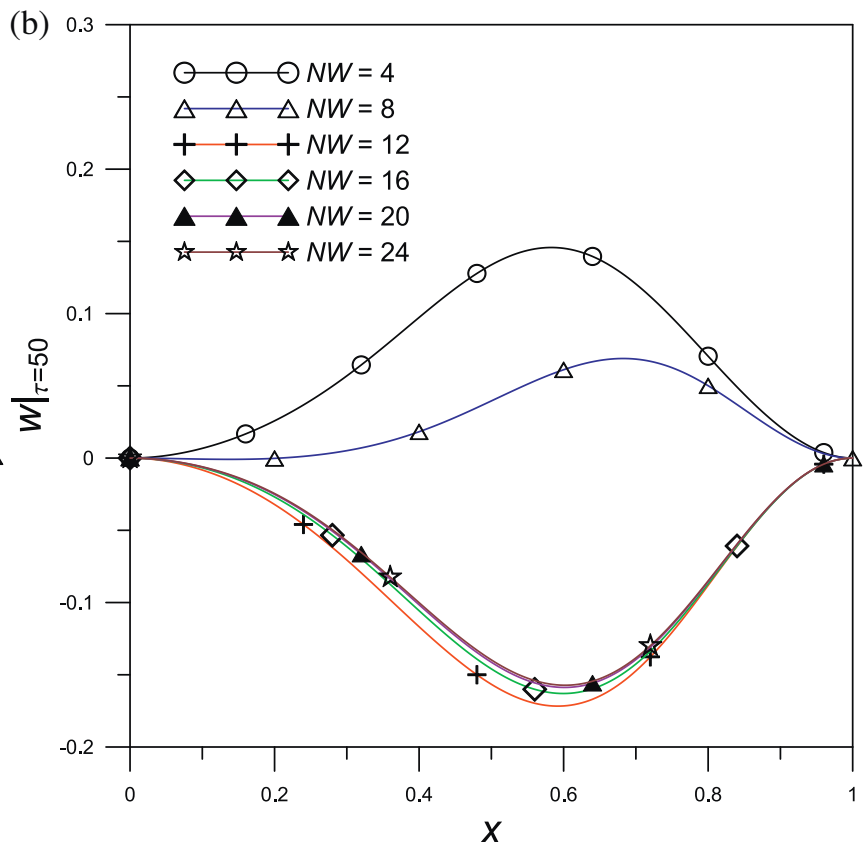

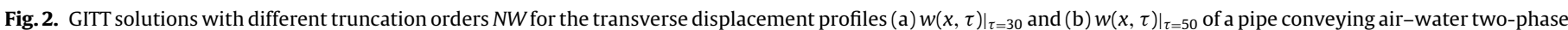
flow. 

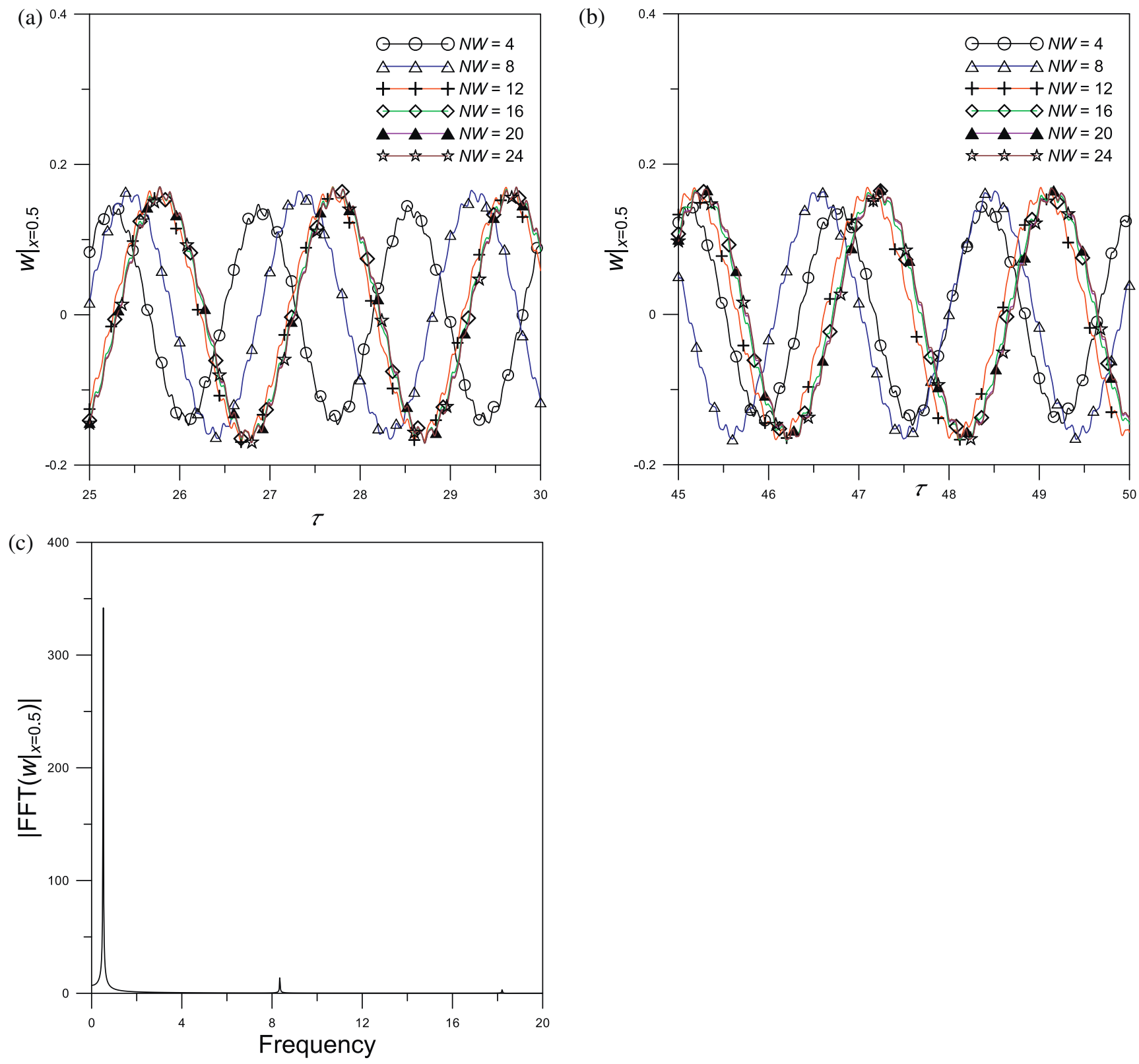

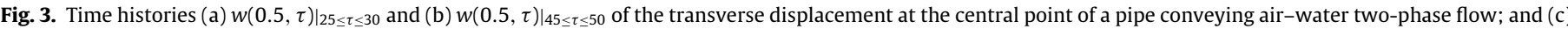
amplitude spectrum of the structural response $\left.w(0.5, \tau)\right|_{0 \leq \tau \leq 50}$.

$$
\begin{aligned}
& \frac{\mathrm{d}^{2} \bar{w}_{i}(\tau)}{\mathrm{d} \tau^{2}}+2 \sum_{k} \Gamma_{k} \beta_{k}^{1 / 2} \sum_{j=1}^{\infty} A_{i j} \frac{\mathrm{d} \bar{w}_{j}(\tau)}{\mathrm{d} \tau}+\mu_{i}^{4} \bar{w}_{i}(\tau) \\
& \quad+\sum_{k} \Gamma_{k}^{2} \sum_{j=1}^{\infty} B_{i j} \bar{w}_{j}(\tau)+\gamma \sum_{j=1}^{\infty} C_{i j} \bar{w}_{j}(\tau) \\
& +\gamma \sum_{j=1}^{\infty} A_{i j} \bar{w}_{j}(\tau)=0, \quad i=1,2,3, \ldots,
\end{aligned}
$$

where the coefficients are analytically determined from the following integrals

$$
A_{i j}=\int_{0}^{1} \tilde{X}_{i}(x) \tilde{X}_{j}(x)^{\prime} \mathrm{d} x
$$

$$
\begin{aligned}
B_{i j} & =\int_{0}^{1} \tilde{X}_{i}(x) \tilde{X}_{j}(x)^{\prime \prime} \mathrm{d} x, \\
C_{i j} & =\int_{0}^{1} \tilde{X}_{i}(x)(x-1) \tilde{X}_{j}(x)^{\prime \prime} \mathrm{d} x .
\end{aligned}
$$

In the similar manner, initial conditions are also integral transformed to eliminate the spatial coordinate, yielding

$\bar{w}_{i}(0)=0, \quad \frac{\mathrm{d} \bar{w}_{i}(0)}{\mathrm{d} t}=v_{0} \int_{0}^{1} \tilde{X}_{i}(x) \sin (\pi x) \mathrm{d} x, \quad i=1,2,3, \ldots$

For computational purposes, the expansion for the transverse deflection is truncated to finite orders NW. Eqs. (13) and (14) in the truncated series are subsequently calculated by the NDSolve routine of Mathematica Wolfram (2003). Once the transformed 


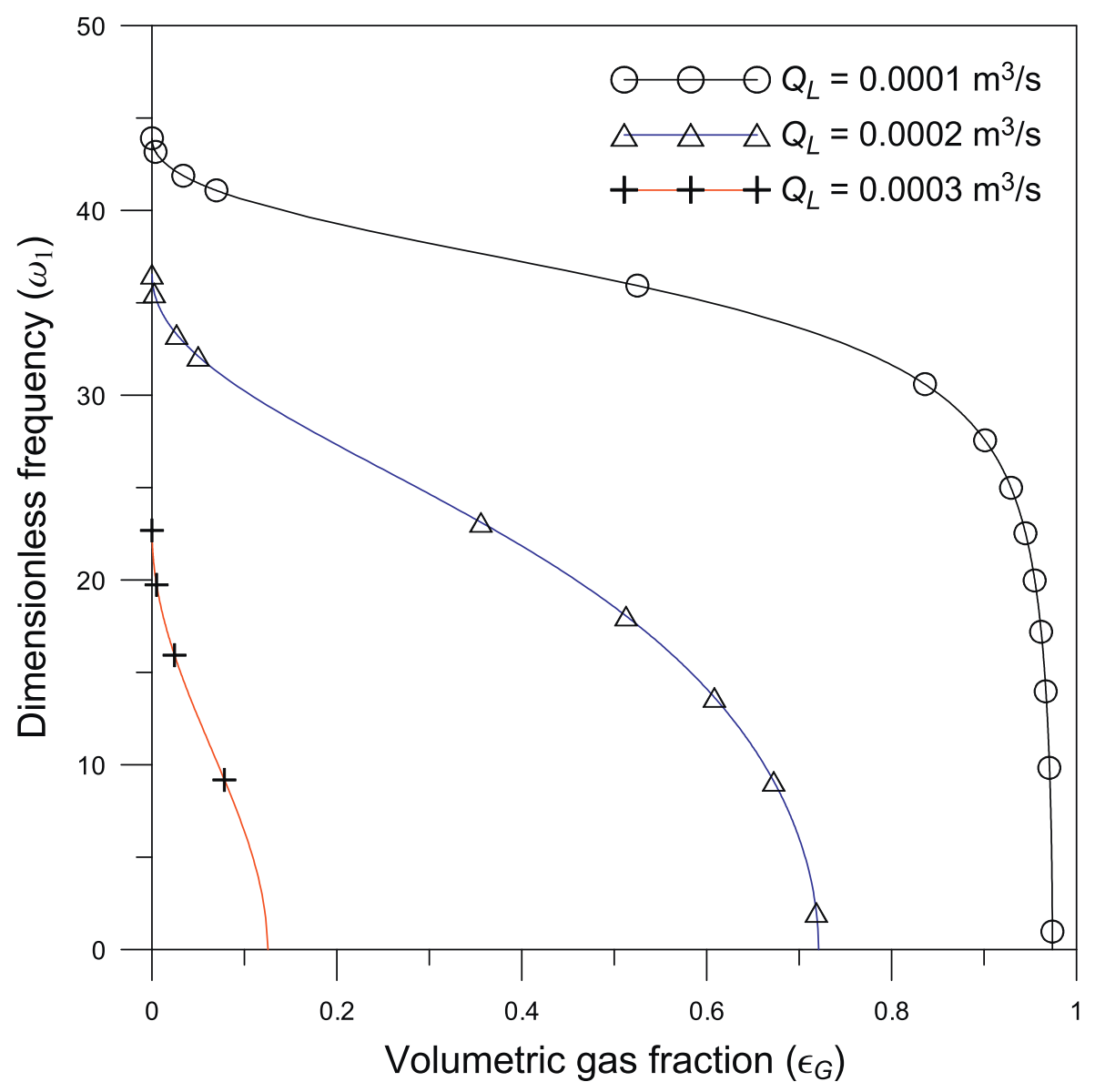

Fig. 4. Variation of the dimensionless frequency $\left(\omega_{1}\right)$ with the volumetric gas fraction $\left(\varepsilon_{G}\right)$.

potential, $\bar{w}_{i}(\tau)$ has been numerically evaluated, the inversion formulas Eq. (12b) is then applied to yield explicit analytical expression for the dimensionless transverse deflection $w(x, \tau)$.

\section{Results and discussion}

\subsection{Two-phase flow model}

Three fundamental parameters of gas-liquid two-phase flow, the volumetric gas fraction $\varepsilon_{G}$, the void fraction $\alpha$, and the slip factor $K$, are defined as follows:

$\varepsilon_{G}=\frac{Q_{G}}{Q_{G}+Q_{L}}$

$\alpha=\frac{A_{G}}{A_{G}+A_{L}}$,

and

$K=\frac{U_{G}}{U_{L}}$,

where $Q_{G}$ and $Q_{L}$ are the volumetric flow rates of the gas and liquid phases, respectively, $A_{G}$ and $A_{L}$ are the area occupied by the gas and the liquid in the inner cross section of the pipe, respectively. The flow velocities of the gas and the liquid are given by

$U_{G}=\frac{Q_{G}}{A_{G}} \quad$ and $\quad U_{L}=\frac{Q_{L}}{A_{L}}$.
The void fraction $\alpha$ is related to the volumetric gas fraction $\varepsilon_{G}$ through the slip factor $K$ :

$\frac{1-\varepsilon_{G}}{\varepsilon_{G}}=\left(\frac{1-\alpha}{\alpha}\right) \frac{1}{K}$.

In this work, the slip factor $K$ is calculated from the model proposed by Monette and Pettigrew (2004):

$K=\left(\frac{\varepsilon_{G}}{1-\varepsilon_{G}}\right)^{1 / 2}$

As pointed out by Monette and Pettigrew (2004), the slip factor $K$ calculated from Eq. (20) is consistent with observed flow patterns and is not very different from Chisholm's slip ratio (Chisholm, 1983).

\subsection{Convergence behavior of the solution}

We now present the convergence behavior of numerical results for the transverse displacement $w(x, \tau)$ of a pipe conveying air-water two-phase flow calculated using the GITT approach. To make the analysis computationally tractable, the parameters of the pipe and the two-phase flow within the parameter range given by Monette and Pettigrew (2004) are taken in Eq. (1). The inner and outer diameters of pipe cross section are $d=12.7 \mathrm{~mm}$ and $D=15.9 \mathrm{~mm}$, respectively. The pipe is considered to be a flexible tube (Tygon ${ }^{\circledR} \mathrm{R}-3606$ ) with the density of $1180 \mathrm{~kg} / \mathrm{m}^{3}$, the Young's modulus of 3.6 MPa and the length of $1 \mathrm{~m}$. For the flow condition, the volumetric air and water flow rates are known: $Q_{G}=0.0005 \mathrm{~m}^{3} / \mathrm{s}$ and $Q_{L}=0.0002 \mathrm{~m}^{3} / \mathrm{s}$ and with the densities of the air and the water $\left(\rho_{G}=1.2 \mathrm{~kg} / \mathrm{m}^{3}\right.$ and $\left.\rho_{L}=1000 \mathrm{~kg} / \mathrm{m}^{3}\right)$, the masses 


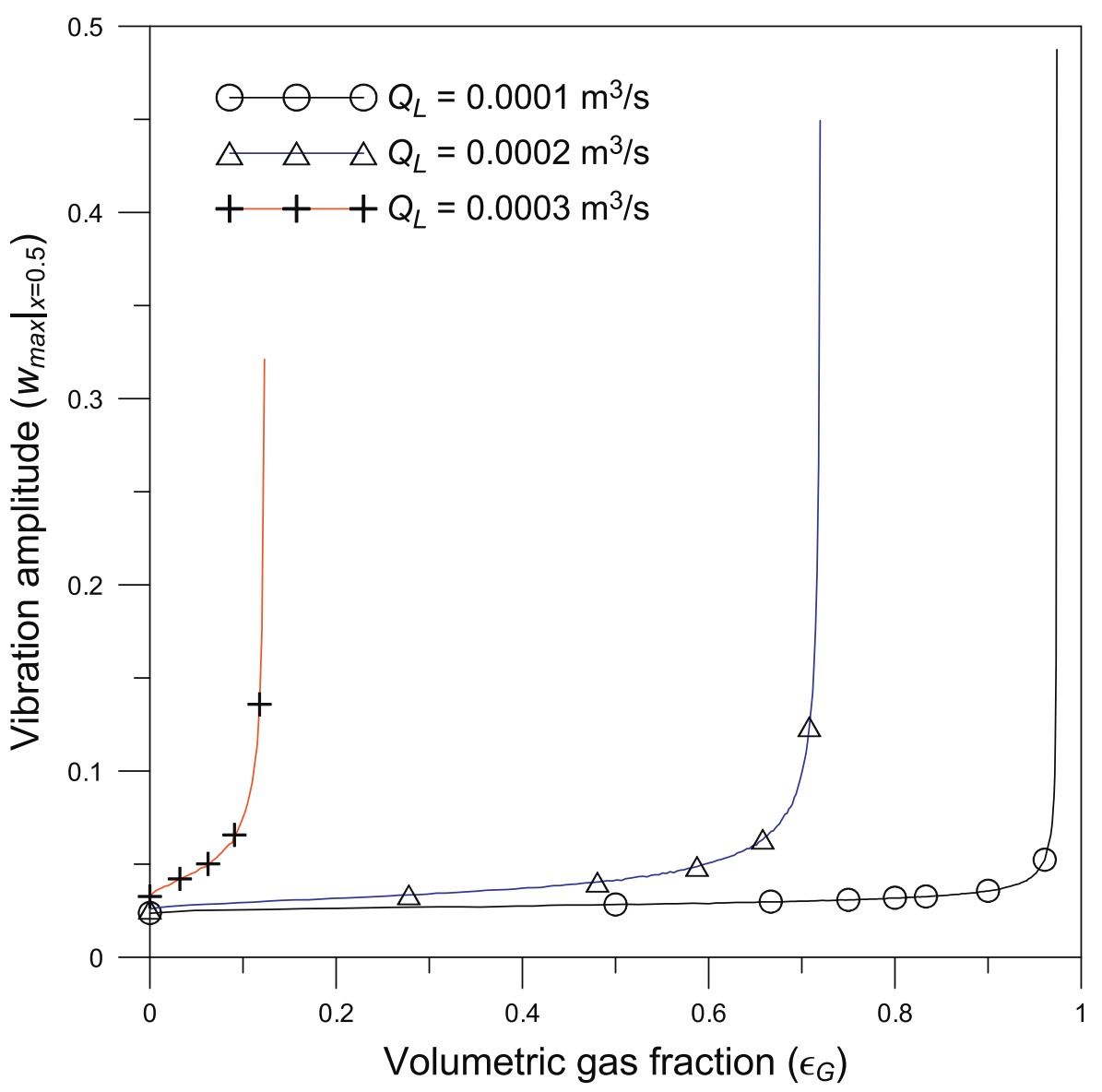

Fig. 5. Variation of the dimensionless vibration amplitude $\left(\left.w_{\max }\right|_{x=0.5}\right)$ with the volumetric gas fraction $\left(\varepsilon_{G}\right)$.

of each phase per unit length can be obtained by $M_{G}=A_{G} \rho_{G}$ and $M_{L}=A_{L} \rho_{L}$. The dimensionless variables can be obtained through Eq. (2). In addition, $v_{0}=1.0$ is employed in the initial conditions (2). The solution of the system, Eqs. (13) and (14), is obtained with $N W \leq 20$ to analyze the convergence behavior.

The dimensionless transverse displacement $w(x, \tau)$ at different positions, $x=0.1,0.3,0.5,0.7$ and 0.9 , of the pipe conveying air-water two-phase flow are presented in Table 1 . The convergence behavior of the integral transform solution is examined for increasing truncation terms $N W=4,8,12,16,20$ and 24 at $\tau=5,20$ and 50 , respectively. Note that the option MaxSteps for NDSolve, which specifies the maximum number of steps that NDSolve will ever take in attempting to find a solution, is set to be $10^{6}$. It can be observed that almost all the solutions converge to the values with two significant digits, while some of them converge to the values with three significant digits with a truncation order of $N W=20$. The results at $\tau=50$ indicate that the convergence behavior of the integral transform solution does not change with time, verifying the good long-time numerical stability of the scheme. For the same cases, the profiles of the transverse displacement at $\tau=30$ and $\tau=50$ are illustrated in Fig. 2 with different truncation orders. In addition, the time histories for $\tau \in[25,30]$ and $\tau \in[45,50]$ of the transverse displacement at the central point of the pipe are shown in Fig. 3 , which also demonstrates that the convergence is achieved at a truncation order of $N W=20$. In order to identify the frequency content of the structural response, the Fast Fourier Transform (FFT) amplitude spectrum of the time history for $\tau \in[0,50]$ of the transverse displacement at $x=0.5$ is shown in Fig. 3(c), from which it can be clearly seen that the three peaks in the amplitude spectrum appear at the frequencies of 0.520 (the fundamental frequency), 8.34 and 18.2 , respectively.

\subsection{Parametric study}

In this section, transverse displacement of pipes conveying air-water two-phase flow with clamped-clamped boundary conditions is analyzed to illustrate the applicability of the proposed approach. The variations of the fundamental frequency $\omega_{1}$ and the vibration amplitude with the volumetric gas fraction $\varepsilon_{G}$ and the liquid flow rate $Q_{L}$ are studied. In the following analysis, we use a relative high truncation order, $N W=20$, for a sufficient accuracy.

\subsubsection{Variation of the fundamental frequency}

To obtain the fundamental circular frequency for the transverse vibration of the system, the coupled ODEs, Eq. (13a), can be represented in the matrix form as follows:

$\mathbf{M} \ddot{\mathbf{w}}(t)+\mathbf{C} \dot{\mathbf{w}}(t)+\mathrm{Kw}(t)=\mathbf{F}(t)$.

Consider the generalized eigenvalue problem of Eq. (21), and the fundamental circular frequency can be obtained by using standard eigenvalue routine for a complex general matrix. The dimensionless fundamental natural frequencies of the pipe conveying air-water two-phase flow for different volumetric gas fractions $0 \leq \varepsilon_{G} \leq 1$ and liquid flow rates $Q_{L}=0.0001,0.0002,0.0003 \mathrm{~m}^{3} / \mathrm{s}$ are calculated, as shown in Fig. 4, where the other geometrical and physical parameters are same as in Section 4.2. It can be seen that the fundamental frequency decreases with the volumetric gas fraction and the liquid flow rate. When $\varepsilon_{G}=0, \omega_{1}$ equal to $43.9,36.6$ and 22.7 for $Q_{L}=0.0001,0.0002$ and $0.0003 \mathrm{~m}^{3} / \mathrm{s}$, respectively, which represent the fundamental frequencies of the pipe conveying liquid. When the volumetric gas fraction reaches a critical value $\left(\varepsilon_{G}=0.974\right.$, 0.721 and 0.125 ), the fundamental frequencies equal to zero for 


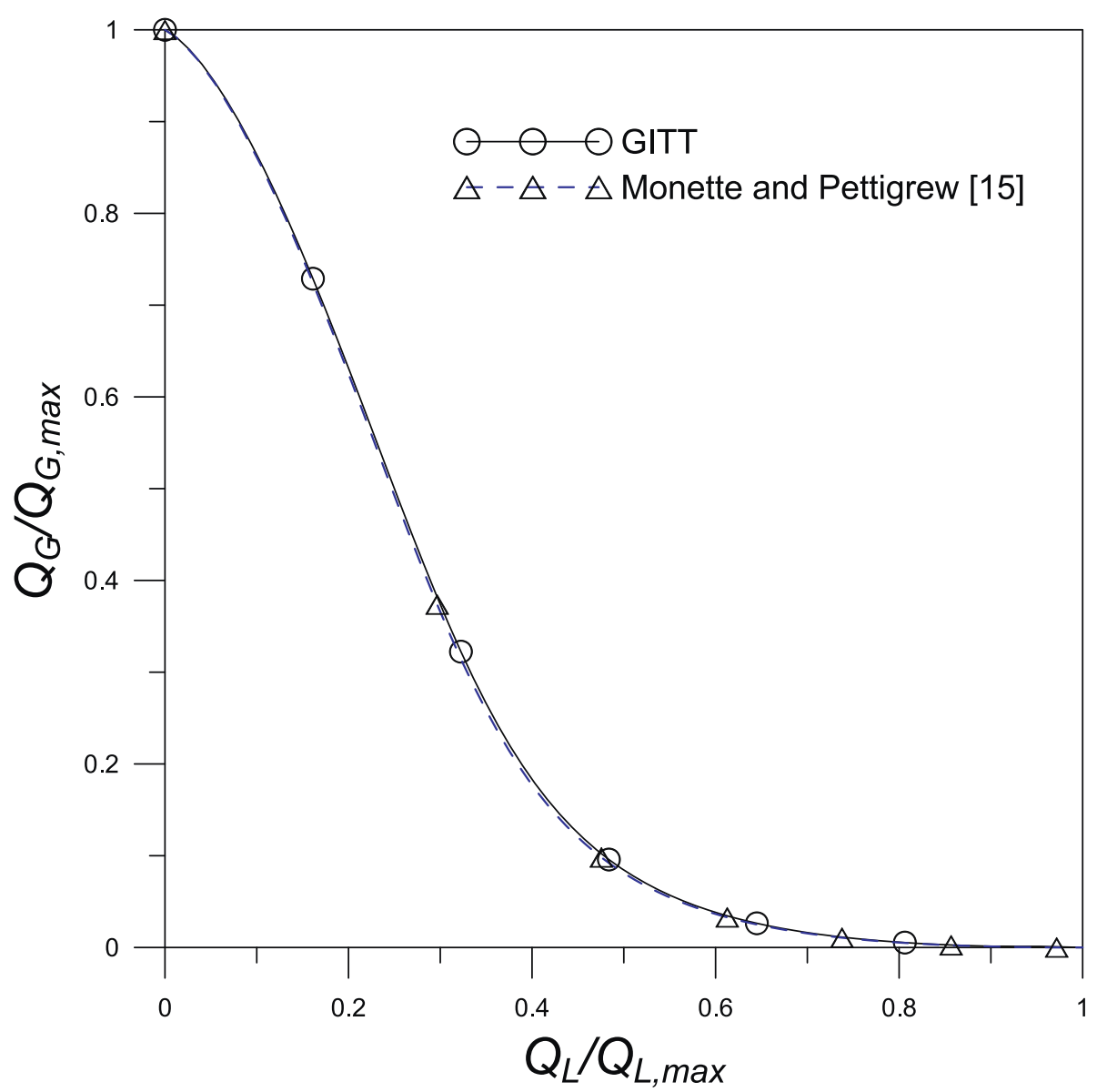

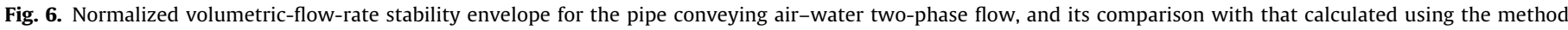
proposed by Monette and Pettigrew (2004).

$Q_{L}=0.0001,0.0002$ and $0.0003 \mathrm{~m}^{3} / \mathrm{s}$, respectively, which means the dynamic system loses its stability by divergence.

\subsubsection{Variation of the vibration amplitude}

The dimensionless vibration amplitudes at the central point of the pipe conveying air-water two-phase flow for different volumetric gas fractions $0 \leq \varepsilon_{G} \leq 1$ and liquid flow rates $Q_{L}=0.0001$, $0.0002,0.0003 \mathrm{~m}^{3} / \mathrm{s}$ are calculated, as shown in Fig. 5, where the other geometrical and physical parameters are same as in Section 4.2. The amplitude is the maximum absolute value found from the calculated time-history response of pipe conveying gas-liquid two-phase flow for $\tau \in[0,50]$. It can be seen that the vibration amplitudes increase with the volumetric gas fraction and the water flow rate. When $\varepsilon_{G}=0,\left.w_{\max }\right|_{x=0.5}$ equal to $0.0237,0.0258$ and 0.0327 for $Q_{L}=0.0001,0.0002$ and $0.0003 \mathrm{~m}^{3} / \mathrm{s}$, respectively, which represent the vibration amplitudes at the central point of the pipe conveying liquid. For each case, the vibration amplitudes tends to infinity (viz., instability of the dynamic system occurs) when the liquid flow rate reaches a critical value, which is the same value given in Fig. 4, proving the coherence of the analysis.

\subsection{Volumetric-flow-rate stability envelope}

When the water flow rate is specified, the critical value of the volumetric gas fraction for the onset of instability of the dynamic system can be calculated as mentioned in Section 4.3.1. Following the same process, the normalized volumetric-flow-rate stability envelope for the pipe conveying air-water two-phase flow can be obtained, as demonstrated in Fig. 6. $Q_{G, \max }=0.00823 \mathrm{~m}^{3} / \mathrm{s}$ and
$Q_{L, \max }=0.000372 \mathrm{~m}^{3} / \mathrm{s}$ represent the critical volumetric gas and liquid flow rates, respectively, when considering the single-phase internal flow. It should be noted that all points located outside the envelope represent a two-phase flow with the specified volumetric gas and liquid flow rates that will cause the dynamic system to lose its stability.

To verify he results given in Fig. 6, the method to calculate the dimensionless critical velocity of each phase and the dimensionless frequency proposed by Monette and Pettigrew (2004) (Section 2. Fluidelastic instability theory in Monette and Pettigrew (2004)) is employed in this work. The classical mode summation response formulation can be expressed as

$w(x, \tau)=\sum_{i=1}^{\infty} a_{i} \tilde{X}_{i}(x) \mathrm{e}^{\mathrm{i} \omega \tau}$.

Then the system of equations, Eq. (13a), is expressed as a summation of modes

$\sum_{j=1}^{\infty}\left[\left(\mu_{j}^{4}-\omega^{2}\right) \delta_{i j}+2 \sum_{k} \Gamma_{k} \beta_{k}^{1 / 2} A_{i j} \omega \mathrm{i}+\sum_{k} \Gamma_{k}^{2} B_{i j}+\gamma C_{i j}+\gamma A_{i j}\right] a_{j}=0$.

To find the critical frequency of the tube at instability, the imaginary part of the frequency term is set to zero. The solution of the system of equations is obtained by allowing the determinant of the coefficients of $a_{j}$ to be equal to zero. In other words, the real and the imaginary part of the determinant are equal to zero, respectively, which yields two equations. By combining the model for slip factor $K$ (Eq. (20)), the three unknowns including the dimensionless velocity of each phase and the dimensionless frequency can be solved. 
The results calculated using the method proposed by Monette and Pettigrew (2004) are also shown in Fig. 6. The excellent agreement between the results obtained and the ones given by GITT indicates the validity of the proposed method in this study.

\section{Conclusions}

The generalized integral transform technique (GITT) has proved in this paper to be a good approach for the analysis of dynamic behavior of pipes conveying gas-liquid two-phase flow, providing an accurate numerical-analytical solution for the natural frequencies and transverse displacements. The solutions confirm good convergence and long-time numerical stability of the scheme. The parametric studies indicate that the fundamental frequency decrease with the volumetric gas fraction and the water flow rate. When the water flow rate reaches a critical value, the dynamic system loses its stability by divergence. The vibration amplitudes increase with the volumetric gas fraction and the water flow rate. In addition, the normalized volumetric-flow-rate stability envelope for the pipe conveying air-water two-phase flow is obtained. The proposed approach can be employed to predict the dynamic behavior of pipes conveying gas-liquid two-phase flow in associated with other more two-phase models for future investigation.

\section{Acknowledgements}

The work was supported by Science Foundation of China University of Petroleum, Beijing (No. 2462013YJRC003 and No. 2462015YQ0403), and by CNPq (Grant No. 306618/2010-9), CAPES and FAPERJ (Grant No. E-26/102.871/2012) of Brazil.

\section{References}

An, C., Su, J., 2011. Dynamic response of clamped axially moving beams: integral transform solution. Appl. Math. Comput. 218 (2), 249-259.

An, C., Su, J., 2014a. Dynamic analysis of axially moving orthotropic plates: integral transform solution. Appl. Math. Comput. 228, 489-507.

An, C., Su, J., 2014b. Dynamic response of axially moving Timoshenko beams: integral transform solution. Appl. Math. Mech., 1-16, http://dx.doi.org/10.1007/s10483014-1879-7

Chen, L.Q., Zhang, Y.L., Zhang, G.C., Ding, H., 2014. Evolution of the double-jumping in pipes conveying fluid flowing at the supercritical speed. Int. J. Non-Linear Mech. 58, 11-21.

Chisholm, D., 1983. Two-phase Flow in Pipelines and Heat Exchangers. George Godwin, Boca Raton, FL.

Cotta, R.M., Mikhailov, M.D., 1997. Heat Conduction - Lumped Analysis, Integral Transforms, Symbolic Computation. John Wiley \& Sons, Chichester, England.

Cotta, R.M., 1993. Integral Transforms in Computational Heat and Fluid Flow. CRC Press, Boca Raton, FL.

Cotta, R.M., 1998. The Integral Transform Method in Thermal and Fluids Science and Engineering. Begell House, New York.

Gu, J.J., An, C., Levi, C., Su, J., 2012. Prediction of vortex-induced vibration of long flexible cylinders modeled by a coupled nonlinear oscillator: integral transform solution. J. Hydrodyn. 24 (6), 888-898.

Gu, J.J., An, C., Duan, M.L., Levi, C., Su, J., 2013. Integral transform solutions of dynamic response of a clamped-clamped pipe conveying fluid. Nucl. Eng. Des. $254,237-245$
Guo, B.Y., Song, S.H., Ghalambor, A., Lin, T.R., 2014. Offshore Pipelines. Design, Installation and Maintenance, second ed. Elsevier, Oxford.

Hara, F., 1977. Two-phase-flow-induced vibrations in a horizontal piping system. Bull. JSME (Jpn. Soc. Mech. Eng.) 20 (142), 419-427.

Huang, Y.M., Liu, Y.S., Li, B.H., Li, Y.J., Yue, Z.F., 2010. Natural frequency analysis of fluid conveying pipeline with different boundary conditions. Nucl. Eng. Des. 240 (3), 461-467.

Kang, M.-G., 2000. The influence of rotary inertia of concentrated masses on the natural vibrations of a clamped-supported pipe conveying fluid. Nucl. Eng. Des. 196 (3), 281-292.

Kuiper, G., Metrikine, A., 2005. Dynamic stability of a submerged, free-hanging riser conveying fluid. J. Sound Vib. 280 (3-5), 1051-1065.

Li, B.H., Gao, H.S., Zhai, H.B., Liu, Y.S., Yue, Z.F., 2011. Free vibration analysis of multispan pipe conveying fluid with dynamic stiffness method. Nucl. Eng. Des. 241 (3), 666-671.

Matt, C.F.T., 2009. On the application of generalized integral transform technique to wind-induced vibrations on overhead conductors. Int. J. Numer. Methods Eng. 78 (8), 901-930.

Matt, C.F.T., 2013a. Combined classical and generalized integral transform approaches for the analysis of the dynamic behavior of a damaged structure. Appl. Math. Modell. 37 (18/19), 8431-8450.

Matt, C.F.T., 2013b. Simulation of the transverse vibrations of a cantilever beam with an eccentric tip mass in the axial direction using integral transforms. Appl. Math. Modell. 37 (22), 9338-9354.

Monette, C., Pettigrew, M., 2004. Fluidelastic instability of flexible tubes subjected to two-phase internal flow. J. Fluids Struct. 19 (7), 943-956.

Ortega, A., Rivera, A., Nydal, O.J., Larsen, C.M., 2012. On the dynamic response of flexible risers caused by internal slug flow. In: Proceedings of the 31st International Conference on Ocean, Offshore and Arctic Engineering, Rio de Janeiro, RJ, Brazil, OMAE2012-83316.

Ortega, A., Rivera, A., Larsen, C.M., 2013. Flexible riser response induced by combined slug flow and wave loads. In: Proceedings of the 32nd International Conference on Ocean, Offshore and Arctic Engineering, Nantes, France, OMAE2013-10891.

Païdoussis, M.P., Li, G.X., 1993. Pipes conveying fluid: a model dynamical problem. J. Fluids Struct. 7 (2), 137-204.

Païdoussis, M.P., 1998. Fluid-Structure Interactions: Slender Structures and Axial Flow. Academic Press, Inc., San Diego, CA.

Païdoussis, M.P., 2008. The canonical problem of the fluid-conveying pipe and radiation of the knowledge gained to other dynamics problems across applied mechanics. J. Sound Vib. 310 (3), 462-492.

Patel, M., Seyed, F., 1989. Internal flow-induced behaviour of flexible risers. Eng. Struct. 11 (4), 266-280.

Pettigrew, M.J., Taylor, C.E., 1994. Two-phase flow-induced vibration: an overview ASME J. Press. Vessel Technol. 116 (3), 233-253.

Pettigrew, M., Taylor, C., Fisher, N., Yetisir, M., Smith, B., 1998. Flow-induced vibration: recent findings and open questions. Nucl. Eng. Des. 185 (2/3), 249-276.

Riverin, J.L., Pettigrew, M.J., 2007. Vibration excitation forces due to two-phase flow in piping elements. ASME J. Press. Vessel Technol. 129 (1), 7-13.

Riverin, J.-L., de Langre, E., Pettigrew, M., 2006. Fluctuating forces caused by internal two-phase flow on bends and tees. J. Sound Vib. 298 (4/5), 1088-1098.

Seyed, F., Patel, M., 1992. Mathematics of flexible risers including pressure and internal flow effects. Mar. Struct. 5 (2/3), 121-150.

Sinha, J.K., Rao, A.R., Sinha, R.K., 2005. Prediction of flow-induced excitation in a pipe conveying fluid. Nucl. Eng. Des. 235 (5), 627-636.

Wolfram, S., 2003. The Mathematica Book, fifth ed. Wolfram Media/Cambridge University Press, Champaign, IL, USA.

Zhai, H.B., Wu, Z.Y., Liu, Y.S., Yue, Z.F., 2011. Dynamic response of pipeline conveying fluid to random excitation. Nucl. Eng. Des. 241 (8), 2744-2749.

Zhang, Y.L., Chen, L.Q., 2012. Internal resonance of pipes conveying fluid in the supercritical regime. Nonlinear Dyn. 67 (2), 1505-1514.

Zhang, Y.L., Chen, L.Q., 2013. External and internal resonances of the pipe conveying fluid in the supercritical regime. J. Sound Vib. 332 (9), 2318-2337.

Zhang, M.M., Xu, J.Z., 2010. Effect of internal bubbly flow on pipe vibrations. Sci. China Technol. Sci. 53 (2), 423-428.

Zhang, M.M., Katz, J., Prosperetti, A., 2010. Enhancement of channel wall vibration due to acoustic excitation of an internal bubbly flow. J. Fluids Struct. 26 (6) 994-1017. 